\title{
Selective control of amino acid metabolism by the GCN2 elF2 kinase pathway in Saccharomyces cerevisiae
}

\author{
John M Zaborske ${ }^{1}$, Xiaochen Wu', Ronald C Wek ${ }^{2}$, Tao Pan ${ }^{1 *}$
}

\begin{abstract}
Background: When eukaryotic cells are deprived of amino acids, uncharged tRNAs accumulate and activate the conserved GCN2 protein kinase. Activated Gcn2p up-regulates the general amino acid control pathway through phosphorylation of the translational initiation factor elF2. In Saccharomyces cerevisiae, Gcn2p is the only kinase that phosphorylates elF2 to regulate translation through this mechanism. We addressed changes in yeast growth and tRNA aminoacylation, or charging, during amino acid depletion in the presence and absence of GCN2. tRNA charging was measured using a microarray technique which simultaneously measures all cytosolic tRNAs. A fully prototrophic strain, and its isogenic gcn2 $\triangle$ counterpart, were used to study depletion for each of the 20 amino acids, with a focus on Trp, Arg, His and Leu, which are metabolically distinct and together provide a good overview on amino acid metabolism.

Results: While the wild-type strain had no observable phenotype upon depletion for any amino acid, the gcn2 $\triangle$ strain showed slow growth in media devoid of only Trp or Arg. Consistent with the growth phenotypes, profiles of genome-wide tRNA charging revealed significant decrease in cognate tRNA charging only in the gcn2 $\triangle$ strain upon depletion for Trp or Arg. In contrast, there was no change in tRNA charging during His and Leu depletion in either the wild-type or gen2 $\triangle$ strains, consistent with the null effect on growth during loss of these amino acids. We determined that the growth phenotype of Trp depletion is derived from feedback inhibition of aromatic amino acid biosynthesis. By removing Phe and Tyr from the media in addition to Trp, regular growth was restored and tRNA $^{\text {Trp }}$ charging no longer decreased. The growth phenotype of Arg depletion is derived from unbalanced nitrogen metabolism. By supplementing ornithine upon Arg depletion, both growth and tRNA ${ }^{\text {Arg }}$ charging were partially restored.

Conclusion: Under mild stress conditions the basal activity of Gcn2p is sufficient to allow for proper adaptation to amino acid depletion. This study highlights the importance of the GCN2 elF2 kinase pathway for maintaining metabolic homeostasis, contributing to appropriate tRNA charging and growth adaptation in response to culture conditions deficient for the central amino acids, tryptophan and arginine.
\end{abstract}

\section{Background}

Translational control is a fundamental step in the regulation of gene expression. An important mechanism for regulating eukaryotic translational initiation involves the phosphorylation of the $\alpha$ subunit of the initiation factor eIF2 in response to environmental stresses [1-3]. The eIF2 delivers initiator tRNA to the translation machinery, and phosphorylation of eIF2 $\alpha$ (eIF $2 \alpha-\mathrm{P})$ reduces it

\footnotetext{
* Correspondence: taopan@uchicago.edu

'Department of Biochemistry and Molecular Biology, University of Chicago, Chicago, IL 60637, USA
}

activity by blocking the eIF2-GDP to eIF2-GTP exchange required for each round of translation initiation. The resulting reduction in global translation conserves energy and allows for cells to reprogram gene expression to ameliorate stress damage. Coincident with this global repression of protein synthesis, eIF $2 \alpha-\mathrm{P}$ elicits preferential translation of selected mRNAs, including the yeast transcriptional activator GCN4 which directs transcription of genes involved in the synthesis of amino acids and the salvaging of nutrients (the socalled general amino acid control, GAAC) [1]. In 
mammals, a related transcription activator, ATF4, is preferentially translated upon increasing eIF $2 \alpha-\mathrm{P}$, contributing to the so-called integrated stress response [4-6].

In the yeast Saccharomyces cerevisiae, Gcn2p is the only known kinase for eIF $2 \alpha$ phosphorylation [1]. Activation of Gcn2p in response to amino acid starvation occurs by Gcn $2 p$ binding to an uncharged tRNA through its histidyl-tRNA-synthetase-like domain [1,7-9]. Although uncharged tRNA ${ }^{\text {His }}$ is known to activate $\operatorname{Gcn} 2 \mathrm{p}$ in vivo, other tRNAs can bind to this HisRS-like domain in vitro [8]. We have shown recently that in vivo, activation of Gcn2p is coincident with enhanced levels of multiple uncharged tRNAs, and the actual uncharged tRNA activator can vary depending on the type of stress [10]. This genome-wide method for measuring tRNA charging uses selective labeling of tRNAs with Cy3 or Cy5 and microarrays spotted with individual probes for each of the yeast tRNAs. Auxotrophic yeast strains were starved for the limiting amino acid, and the charging levels for both the cognate tRNA and some non-cognate tRNAs were decreased. These key results were confirmed by northern analyses, validating the method and the key findings. These results suggest that activation of Gcn2p occurs in response to a complex pattern of changes in tRNA charging upon starvation for amino acids.

In addition to the GAAC, cells use other mechanisms to regulate the activity of various metabolic pathways. For the aromatic amino acid biosynthetic super-pathway, the first step, biosynthesis of 3-deoxy-darabinoheptulosonate-7-phosphate, is under the control of both GCN4 and feedback inhibition of metabolites [11]. While GCN4 can upregulate the expression of biosynthetic enzymes, the availability of Tyr and Phe can modulate the enzymatic activity of this step. To control arginine metabolism and the urea cycle, cells elicit transcriptional control by Gcn4p, along with monitoring the intracellular concentration of arginine, ornithine and citrulline to fine tune metabolic flux $[12,13]$. In this study, we investigated the mechanisms by which yeast cells respond to individual amino acid depletion in a GCN2 dependent manner by monitoring cell growth and genome-wide tRNA charging levels. Our results reveal a complex relationship between cellular metabolism and GCN2 function.

\section{Results \\ GCN2-dependent recovery of cell growth upon amino acid depletion}

Yeast can synthesize each of the twenty amino acids. To screen for conditions in which the GCN2 pathway is required for prototrophic yeast cells to recover from amino acid depletion, we systematically depleted one amino acid from the medium for each of the 20 amino acids. Yeast cells were first grown to saturation in SC medium supplemented with all 20 amino acids. Cells were then pelleted and resuspended to $\mathrm{A}_{600} \sim 0.1$ in the same medium containing all 20 or just 19 amino acidsabsent a selected depleted amino acid. Cell growth was monitored in microplates for $24 \mathrm{~h}$ (Fig. 1A). To determine the requirement for GCN2 during the nutrient shift, growth of a prototrophic strain containing an intact GAAC pathway was compared to an isogenic $g c n 2 \Delta$ strain. The wild-type GCN2 strain showed no significant growth differences during these drop-out media conditions. By comparison, the gcn $2 \Delta$ strain showed no growth phenotype upon depletion for each of 18 amino acids; however, depletion of tryptophan or arginine showed a strong growth defect. To address whether depletion for Trp or Arg specifically blocked growth during the transition into early-log growth, we depleted these two amino acids in flasks after cells were grown to the mid-logarithmic phase (Fig. 1B). The same growth defect was observed, indicating that GCN2 is required in response to depletion for either Trp or Arg in SC medium. Using a GCN4-lacZ reporter, we show that the Gcn2p dependent translational regulation of the GCN4 mRNA is indeed drastically reduced in the gcn $2 \Delta$ strain before and after depletion of Arg (Fig. 1C, [14]).

\section{GCN2-dependent tRNA charging effects upon amino acid depletion}

Since elevated levels of uncharged tRNAs are central to the activation of Gcn2p and the GAAC, we applied a tRNA microarray method to measure the charging level of all tRNAs simultaneously ([10], Fig. 2). Total charged tRNAs were isolated from cells under mildly acidic conditions to retain the amino acid attached to the $3^{\prime}$ end. The total RNA sample was then split into two portions, one half was treated with periodate which only oxidizes the 3' end of uncharged tRNAs - this half measures the amount of charged tRNAs. The other half was treated with buffer only - this half measures the amount of total tRNAs. Both halves were deacylated at alkaline $\mathrm{pH}$, and Cy3 and Cy5 fluorophore containing oligonucleotides were ligated onto only those tRNAs with intact 3 ' ends using T4 DNA ligase. After labeling, samples with opposite fluorophores (e.g. charged tRNA with Cy5 and total tRNA with Cy3) were combined and hybridized on the same microarray.

Both tryptophan and arginine depletion are known to cause growth defects in $g c n 4 \Delta$ and the growth rate analysis confirmed that our gcn $2 \Delta$ strain exhibited this phenotype as well ([15-17], Fig. 1). We chose to examine the changes in the tRNA charging profile upon depletion of four amino acids: Trp (Fig. 2A, B) and Arg (Fig. 2C), which showed significant growth defects in the $g c n 2 \Delta$ cells, and Leu and His (Fig. 2D), which have 

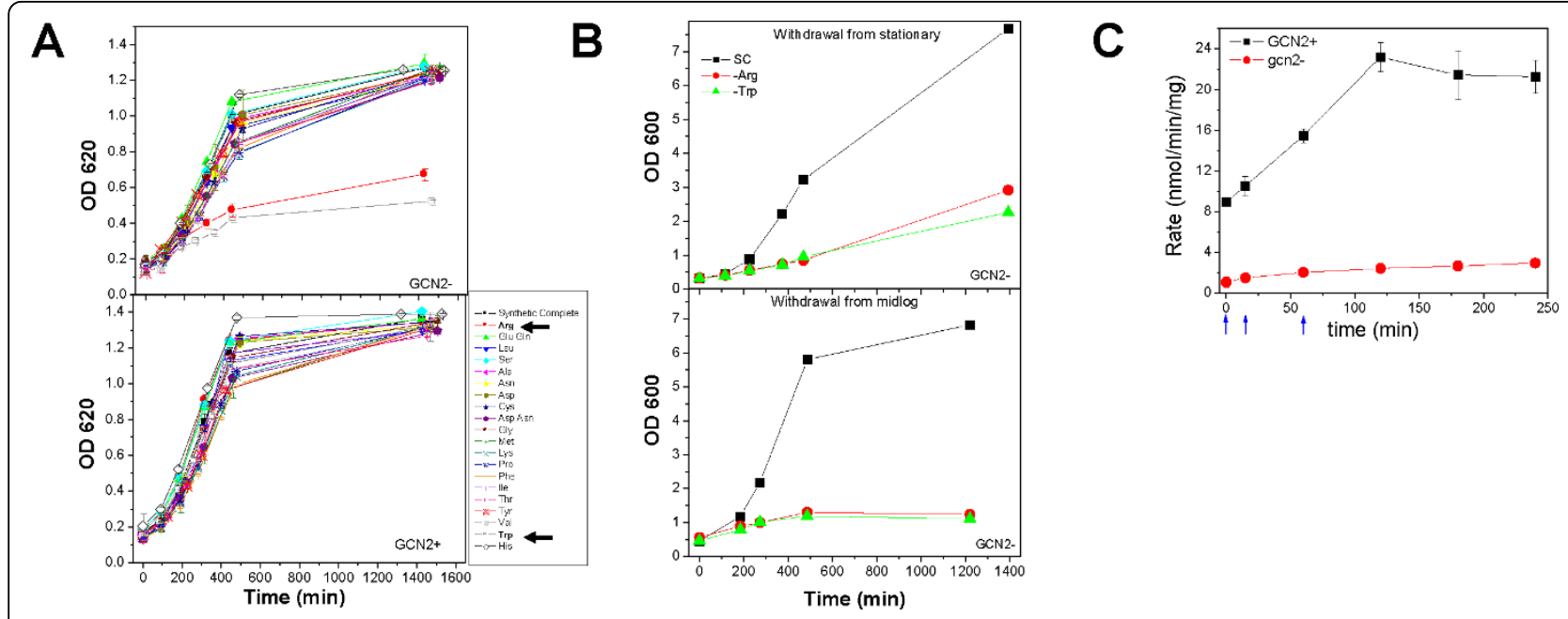

Figure 1 Yeast growth upon single amino acid depletion shows recovery of Trp and Arg depletion dependent on GCN2. (A) Systematic depletion of amino acids from growth media in 96 well plates. Arrows indicate slow growth in Arg and Trp in the gcn2 $\triangle$ strain. (B) Recovery of growth in flasks after amino acid depletion. Depletion occurred while cells were in stationary or mid log growth. (C) Measurements of the GCN4lacZ reporter showed that the Gcn2p-dependent translational regulation of the GCN4 mRNA is significantly reduced in the gcn2 $\triangle$ strain before and after Arg depletion. This result suggests that the level of the GCN4 protein is drastically lower in the gcn $2 \Delta$ strain as expected. Blue arrows indicate time points at which tRNA charging profiles were measured.

extensive but distinct anabolic and catabolic pathways from Trp and Arg. The tRNA charging results showed a significant correlation to the growth profiles for each of these amino acids. Upon Trp depletion, the charging levels of $\mathrm{tRNA}^{\mathrm{Tr}}$ were lowered by $\sim 2$-fold at $15 \mathrm{~min}$ for both strains. By $60 \mathrm{~min}$, tRNA ${ }^{\text {Trp }}$ charging did not decrease further in the wild-type cells, but declined by 10 -fold in the gcn $2 \Delta$ strain (Fig. 2A). This very large decrease of $\mathrm{tRNA}^{\mathrm{Tr}}$ charging does not correlate with a significant change in the RNA $^{\text {Trp }}$ levels under the same condition (Fig. 2B). Aminoacylation of the initiator $\mathrm{tRNA}_{\mathrm{i}}{ }^{\text {Met }}$ was also selectively reduced in the $g c n 2 \Delta$ strain in response to removal of Trp. It is unclear why Trp depletion leads to selective decrease in initiator $\mathrm{tRNA}_{\mathrm{i}}{ }^{\text {Met }}$ charging. However, reduced charging level of initiator $\mathrm{tRNA}_{\mathrm{i}}{ }^{\text {Met }}$ makes biologically sense because this would lead to decreasing the global level of translation and furthermore to up-regulation of GCN4 translation and enhanced GAAC [18]. Upon Arg depletion, the charging levels of all four tRNA ${ }^{A r g}$ isoacceptors were reduced by $\sim 2$-fold at $15 \mathrm{~min}$ and $\sim 10$-fold at $60 \mathrm{~min}$, but only in the gcn $2 \Delta$ strain. The charging of several non-cognate tRNAs, $\mathrm{tRNA}^{\text {Glu }}$ and $\mathrm{tRNA}^{\text {Asp }}$ and to a lesser extent $\mathrm{tRNA}^{\mathrm{Lys}}$ and $\mathrm{tRNA}{ }^{\mathrm{Tyr}}$, were also lowered in the $g c n 2 \Delta$ strain upon Arg depletion in the medium. Therefore, in both cases of Trp and Arg depletion, the growth defect of the $g c n 2 \Delta$ strain is accompanied by dramatic charging reductions in cognate tRNAs. The control experiments involving Leu and His depletion showed no appreciable decrease in the charging level of any tRNA in either wild-type or gcn $2 \Delta$ strains, consistent with the null effect of these two amino acid depletions on cell growth.

To further examine the quantitative dependence and dynamics of tRNA charging upon Trp and Arg depletion in the $g c n 2 \Delta$ strain, we examined the effect of partial Trp and Arg depletion in the SC medium (Fig. 3). When all other amino acids are present in the medium, partial Trp depletion showed no change in growth until the Trp concentration was at or below $6 \mu \mathrm{g} / \mathrm{ml}$ (Fig. $3 \mathrm{~A})$. $\mathrm{tRNA}^{\mathrm{Trp}}$ charging levels mirrored this result, as there was no significant decrease in $\mathrm{tRNA}^{\text {Trp }}$ charging until the Trp concentration was at this same level. The time-dependent drop of tRNA ${ }^{\text {Trp }}$ charging was gradual and the time required to reduce $\mathrm{RNA}^{\mathrm{Tr}}$ charging by $50 \%$ was $\sim 25 \mathrm{~min}$ (Fig. 3A). The graded depletion of Arg from the SC medium also showed selective reductions in the growth of the gcn $2 \Delta$ cells, where a threshold of $6 \mu \mathrm{g} / \mathrm{ml}$ elicited a significant reduction compared to the SC medium sated with Arg (Fig. 3B). The tRNA ${ }^{\text {Arg }}$ charging levels also reflected this result on growth. The time-dependent drop of $\mathrm{tRNA}^{\text {Arg }}$ charging was $\sim 10 \mathrm{~min}$ for $50 \%$ reduction of $t R N A^{A r g}$ charging levels (Fig. 3B). These results indicate that in the absence of GCN2, the charging levels of tRNA ${ }^{\text {Trp }}$ and tRNA ${ }^{\text {Arg }}$ and cell growth are sensitive to the concentration of these two crucial amino acids in the media.

\section{Cellular pathway linked to Trp sensitivity}

In order to better understand why Trp depletion is deleterious to cells lacking GCN2, we focused on tryptophan metabolism. Biosynthesis of aromatic amino acids is 


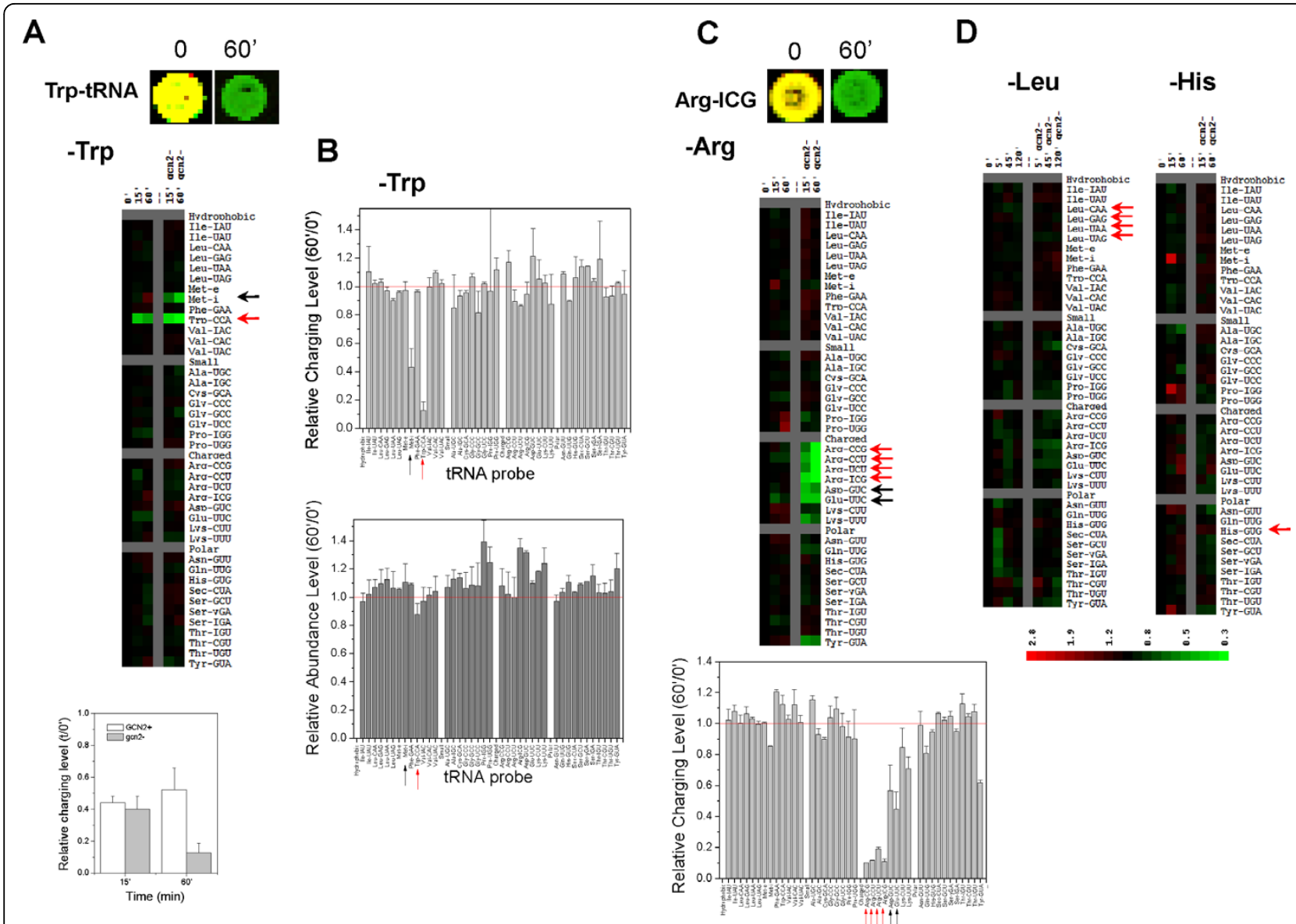

Figure 2 Changes in tRNA charging profile correlate with growth phenotype. Cognate tRNAs for the amino acid depleted are indicated by red arrows. Non-cognate tRNAs whose charging also changes significantly are indicated by black arrows. (A) Trp depletion. Top: array spot for the Trp-tRNA probe; Middle: heatmap of the wild-type and isogenic gcn2 $\triangle$ strains; Bottom: histogram of the relative charging level of tRNA ${ }^{T r p}$ before and after Trp depletion. (B) Comparison of the changes in tRNA charging (top) and tRNA abundance (bottom) before and after 60 min of Trp depletion of the gen2 $\triangle$ strain. Both tRNA ${ }^{\text {Trp }}$ and initiator tRNA ${ }_{i}^{\text {Met }}$ exhibited a large decrease in charging, but not in abundance. (C) Arg depletion. Top: array spot for the Arg-tRNA (anticodon ICG) probe; Middle: heatmap of the wild-type and isogenic gcn2 $\triangle$ strains; Bottom: histogram of the relative charging level before and after $60 \mathrm{~min}$ of Arg depletion for the gcn $2 \Delta$ strain. (D) Leu and His depletion showed no significant decrease in tRNA charging.

subject to feedback inhibited and can be activated at multiple steps; however, the most central step for this work is the feedback inhibition at the first reaction of this pathway (Fig. 4A, [19]). This first reaction can be down regulated by an excess of phenylalanine and tyrosine. Additionally, the genes encoding many of these biosynthetic enzymes are transcriptionally regulated by GCN4, including ARO3 and ARO4, which participate in the first step of the pathway leading to the synthesis of chorismate [20,21]. We hypothesized that knocking out GCN2 eliminates the ability of the cell to properly regulate aromatic amino acid biosynthesis. When Trp alone was depleted, cellular Trp synthesis cannot proceed normally in the absence of Gcn2p because large amounts of Phe and Tyr are still available in the medium. These two amino acids would then inhibit the first step of this biosynthesis super-pathway, thereby inhibiting the proper synthesis of Trp.

This hypothesis posits that depleting all three aromatic amino acids simultaneously could restore growth in the $g c n 2 \Delta$ cells; this was indeed observed (Fig. 4B). The tRNA charging profile after the simultaneous removal of three aromatic amino acids (Fig. 4C, D) showed that restoration of the growth phenotype in the $g \operatorname{cn} 2 \Delta$ strain is accompanied by the maintenance of high charging levels of tRNA ${ }^{\mathrm{Trp}}$ at all times upon Trp/Phe/Tyr depletion, while charging for tRNA ${ }^{\text {Phe }}$ and tRNA $^{\text {Tyr }}$ showed modest decrease. These results indicate that the requirement for GCN2 for growth and maintenance of tRNA charging in the SC medium limiting for Trp is both a consequence of the impaired GAAC and feedback inhibition regulating the carbon flux through the 

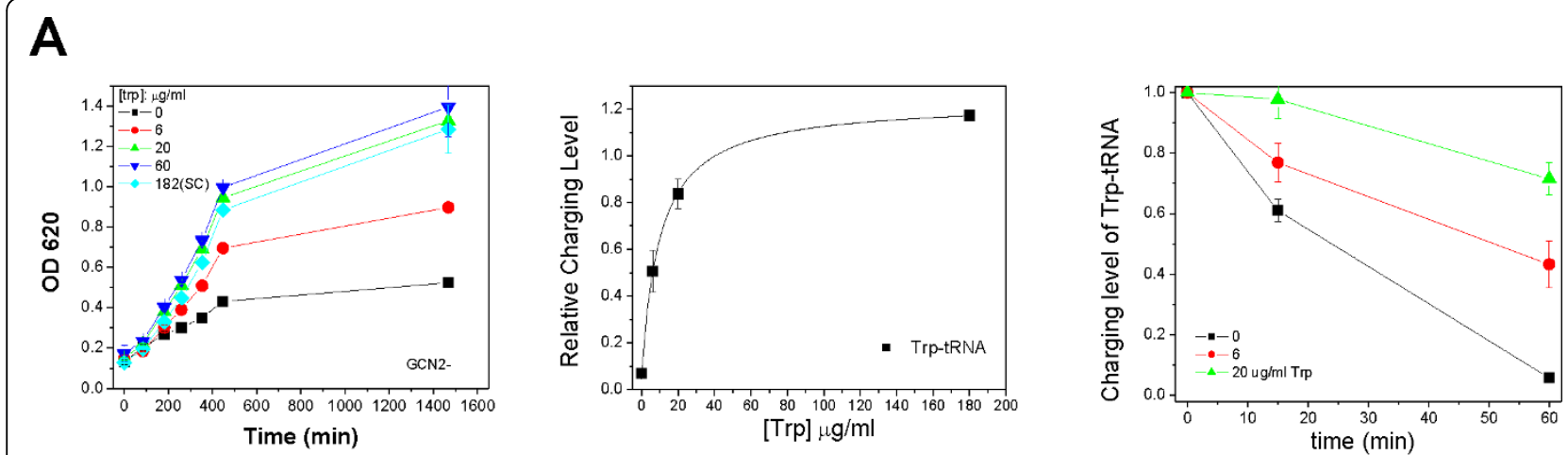

B
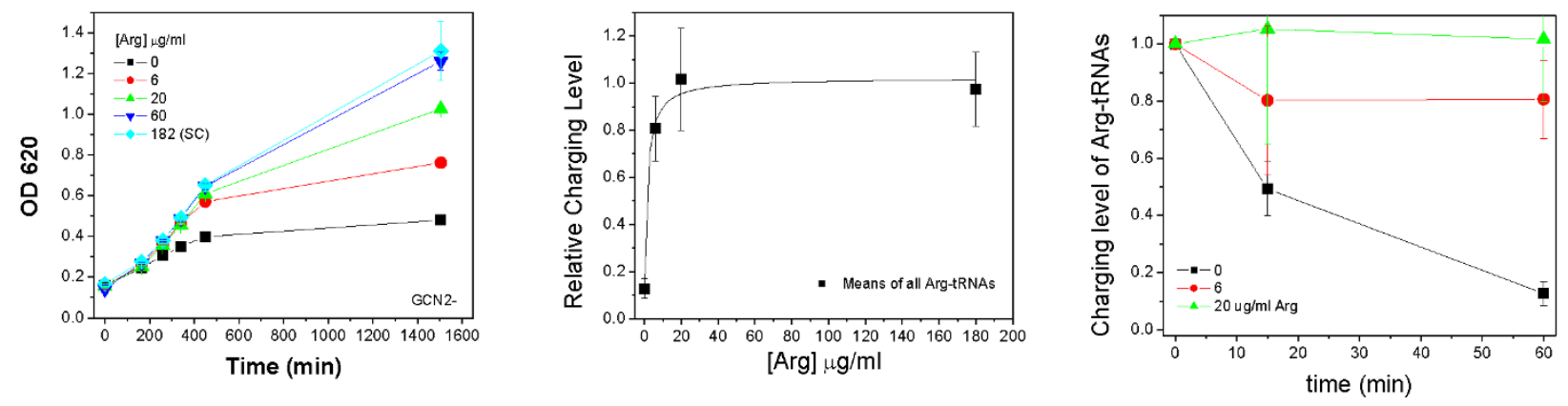

Figure 3 Partial Trp and Arg depletion of the gen $2 \Delta$ strain shows graded sensitivity of Trp and Arg availability to Gcn2p. The complete media contain $182 \mu \mathrm{g} / \mathrm{ml}$ Trp and $182 \mu \mathrm{g} / \mathrm{ml}$ Arg. (A) Trp. Growth at varying Trp concentrations (left), the tRNA ${ }^{\text {Trp }}$ charging levels 60 min after depletion (middle), time course of tRNA $A^{\text {Trp }}$ charging at various Trp concentration in the media (right). (B) Arg. Growth at varying Arg concentrations (left), the averaged charging levels of all four tRNA ${ }^{\text {Arg }}$ isoacceptors 60 min after depletion (middle), time course of the averaged charging levels of all four tRNA ${ }^{\text {Arg }}$ isoacceptors at various Arg concentration in the media (right).

biosynthetic pathway for aromatic amino acids. Depletion of Trp alone retains Tyr and Phe in the SC medium which then can inhibit this combined synthesis pathway. Because Tyr and Phe are readily available in the SC medium depleted of Trp alone, cells should import sufficient amounts of Tyr and Phe to maintain the charging levels of tRNA ${ }^{\text {Phe }}$ and tRNA ${ }^{\text {Tyr }}$.

\section{Cellular pathway linked to Arg sensitivity}

In order to better understand why Arg depletion is deleterious to cells lacking GCN2, we focused on cellular utilization of arginine. Many genes in the arginine biosynthesis are transcriptionally regulated by GCN4. Arg is not only important for protein synthesis but is also a necessary precursor for the synthesis of other metabolites. For example, as a part of the urea cycle, Arg is the immediate precursor to ornithine, which is necessary to make citrulline and polyamines (Fig. 5A, [22]). To address the importance of these metabolic relationships in growth phenotype of the $g c n 2 \Delta$ cells in Arg-deficient medium, we depleted arginine from the SC media, but supplemented this medium with ornithine or citrulline (Fig. 5B). Growth was partially restored upon ornithine supplementation but not with citrulline. The addition of ornithine also partially alleviated the tRNA ${ }^{\text {Arg }}$ charging defect as compared to the absence of ornithine (Fig. 5C, D). Charging of all tRNA ${ }^{\text {Arg }}$ isoacceptors was essentially unchanged in the $g c n 2 \Delta$ cells after 15 minutes of Arg depletion, and showed a $\sim 2$-fold decrease only after 60 minutes, whereas the charging level of tRNA ${ }^{\text {Arg }}$ was reduced by 5 -10-fold in the absence of ornithine.

Ornithine is only used as a metabolic precursor for citrulline and polyamine biosynthesis. It may be possible that supplementing media with citrulline and polyamines in the absence of arginine would recover growth in a manner similar to ornithine supplementation. However, no growth recovery was observed when citrulline and polyamines were added after arginine depletion (Fig. $5 B)$. Increased concentrations of other nitrogen sources such as Glu and Gln upon Arg depletion also failed to rescue growth (data not shown). These results indicate that aside from Arg, ornithine is unique in this pathway in balancing nitrogen metabolism in the absence of GCN2.

\section{Discussion}

The GAAC pathway is essential for regulating metabolism especially when cells are subjected to nutrient 


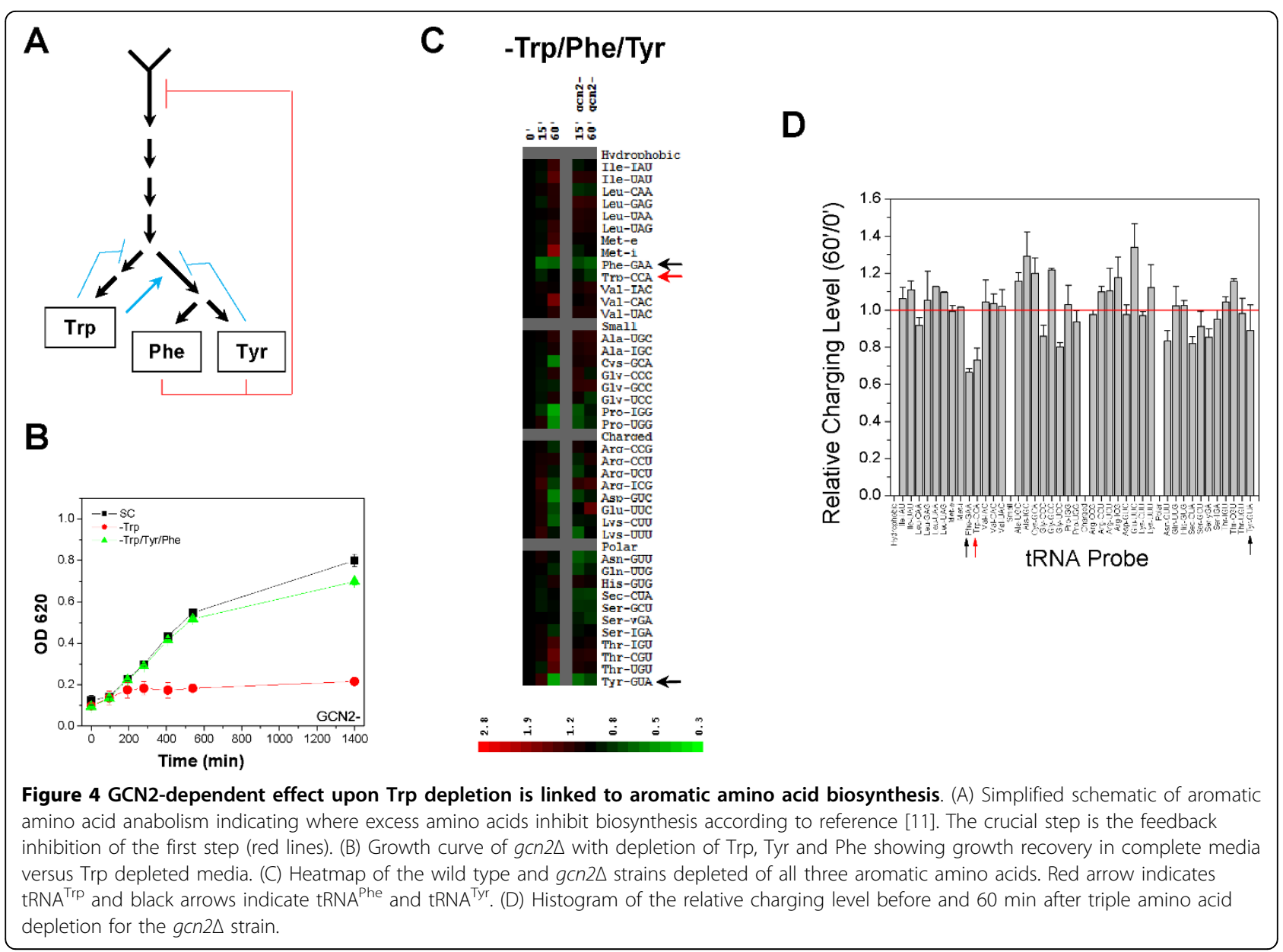

stresses. Previous work indicated that activation of Gcn2p in auxotrophic strains produces surprising tRNA charging profiles which can activate Gcn2p and consequently GAAC [10]. In this work we focus on how Gcn2p is used to regulate metabolic flux in prototrophic cells.

While many stresses are known to activate Gcn2p, mild stresses of prototrophic $S$. cerevisiae, such as depleting a single amino acid from the growth media as done in this work do not require activation of Gcn2p under all conditions. One possible reason for this selective response to amino acid availability is that many metabolic pathways contain alternative regulators, in addition to GCN4 $[23,24]$. For example, Chin et al. [25] showed that during the mild nutrient stress of leucine depletion, Leu3p can specially regulated the Leu biosynthetic pathway, and can sufficiently regulate the branched aromatic amino acid biosynthesis super-pathway independent of GCN4 upregulation. Our results agree nicely with this previous study and furthermore, we observed no change in charging of any tRNA upon Leu depletion.
The first step of the aromatic amino acid biosynthetic super-pathway uses feedback inhibition in combination with GCN4 for regulation instead of using two separate transcription factors as in Leu biosynthesis. Our results confirm that improper regulation of this super-pathway causes growth and tRNA charging defects in gcn $2 \Delta$ cells subjected to tryptophan depletion. This may be explained by the $g c n 2 \Delta$ strain lacking a non-GCN4 protein regulator of the first step of the aromatic amino acid biosynthetic pathway. Lack of Gcn2p mediated upregulation of GCN4 ultimately results in an auxotrophlike phenotype. What would happen to the growth of $g c n 2 \Delta$ strain if both Tyr and Phe are depleted? Addition of Tyr and Phe to a yeast strain in which the GCN2 pathway was genetically inactivated did not provide for growth, whereas the addition of Tyr alone or Phe alone did promote growth [15-17]. However, the addition of Trp alone in this yeast strain fully restored growth. These results suggest that as long as Trp is present, double depletion of Tyr and Phe would confer no effect on growth in $g c n 2 \Delta$ strains. 


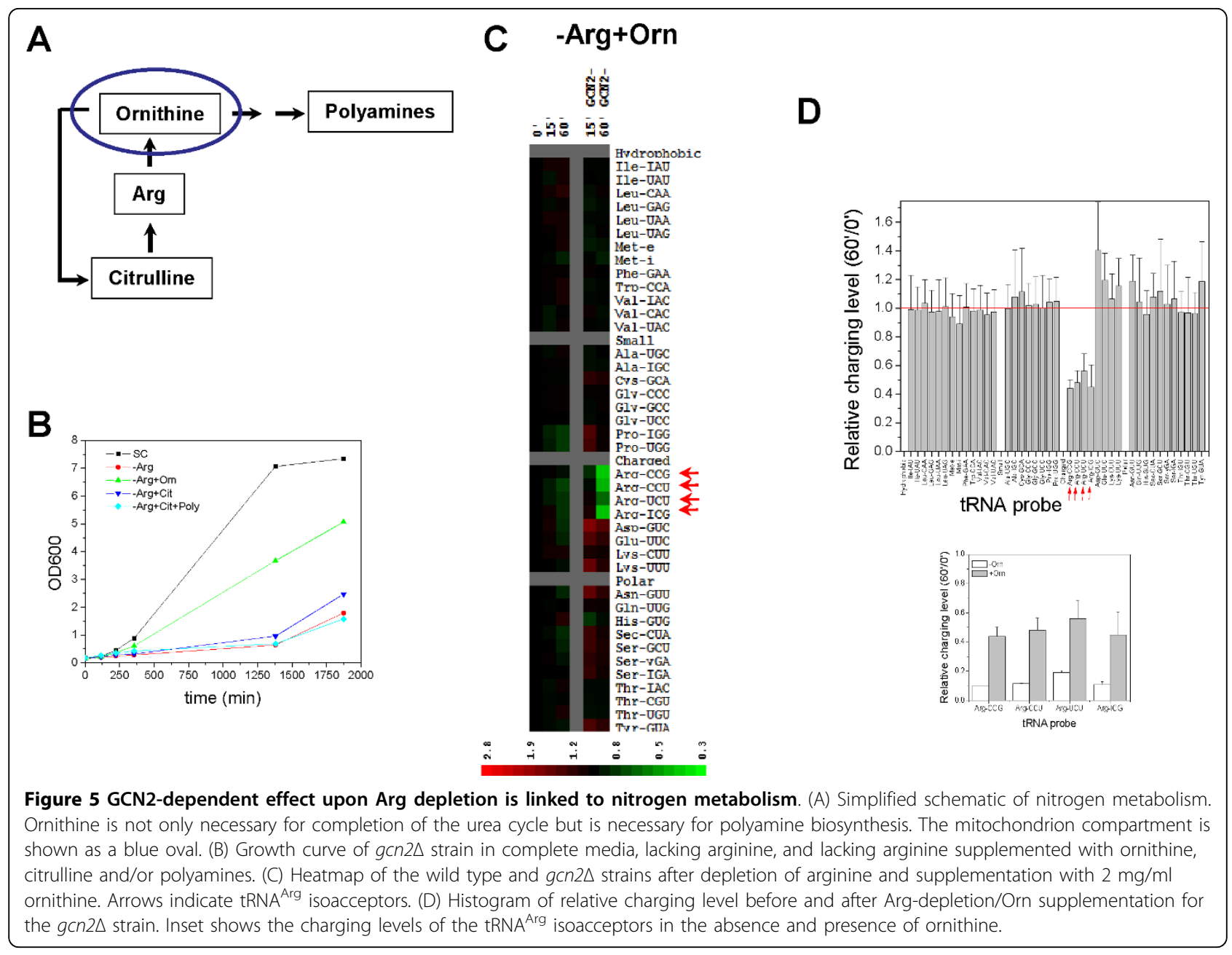

Arginine metabolism is also regulated by feedback inhibition of metabolites and by the transcription factor Gcn4p. Unlike tryptophan biosynthesis, arginine metabolism is not strictly a linear pathway. Both arginine anabolism and catabolism are linked to the urea cycle. In the case of the urea cycle, cell uses the available arginine, ornithine, and citrulline to effect enzymatic activity in addition to Gcn4p to regulate metabolic flux $[13,26]$. While we were unable to completely eliminate the growth defect in $g c n 2 \Delta$ strains with supplements, partial growth restoration was possible in media containing high concentrations of ornithine. This result may be related to ornithine transport across cell membranes. Inefficient uptake from the media after arginine depletion could result in disrupted nitrogen flux. Another possibility is that ornithine, a known inhibitor of arginase activity in vitro [27], acts as an inhibitor of arginase which converts arginine to ornithine and releases urea as a byproduct. Therefore, it is possible that ornithine may accumulate in the cytosol and inhibit arginase. In this way, the presence of ornithine in the cytosol would reduce the conversion of arginine to ornithine, leaving more arginine available for translation.

We also observed decreased charging levels of specific tRNAs that are not cognate to the depleted amino acid, e.g. initiator $\mathrm{tRNA}_{\mathrm{i}}{ }^{\mathrm{Met}}$ upon Trp depletion or tRNA ${ }^{\mathrm{Asp}}$ and tRNA ${ }^{\text {Glu }}$ upon Arg depletion. In the genetic background used here $(g \operatorname{cn} 2 \Delta)$, a possible cause of these changes in tRNA charging is derived from regulated expression levels in their cognate aminoacyl-tRNA synthetases prior to amino acid depletion. Transcription of at least one aminoacyl-tRNA synthetase is known to be up-regulated by GCN4 [28]. Hence, the extremely low level of GCN4 protein in the $g c n 2 \Delta$ strain (Fig. 1C) could reduce the expression of certain tRNA synthetases, thereby predisposes these cells to become hypersensitive to metabolic fluxes upon amino acid depletion.

\section{Conclusions}

In summary, this work examines growth and genomewide tRNA charging response in wild type and $g c n 2 \Delta$ cells subjected to mild nutritional stress. Although eIF2 
phosphorylation was thought to control translation upon the depletion of any amino acid, we found that this conserved mechanism of translational control is particularly sensitive to the availability of just two amino acids, Trp and Arg. This GCN2 dependent response to the limitation of Trp and Arg is due to synthesis of Trp and degradation of Arg. Our results show unexpected complexity of cellular metabolism and tRNA charging, and indicate that some metabolic pathways are particularly sensitive to translational control.

\section{Methods}

\section{Cell strains and growth}

The $S$. cerevisiae strains used in this study were derived from EG328-1A (MATa ura3-52 leu2 trp1) [29,30]. Strain EG328-1A was transformed with DNA fragments containing the LEU2, TRP1, or URA3 genes, generating a prototrophic strain, WY798 (MAT $\alpha$ URA3 LEU2

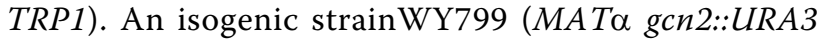
LEU2 TRP1) contains a deletion in the GCN2 gene. Cells were grown overnight in filter sterilized synthetic complete (SC) media containing 2\% dextrose, $0.5 \%$ ammonium sulfate, and all amino acids [31]. To screen for growth in media with selected amino acids, cells were grown overnight to saturation $\left(\mathrm{A}_{600} \sim 6-7\right)$ in $\mathrm{SC}$ media containing all amino acids. Cells were collected by centrifugation, and then resuspended in SC media lacking only the specified amino acid to $\mathrm{A}_{600}$ of $\sim 0.1$. Cells were grown in 96 well plates at $30^{\circ} \mathrm{C}$ with constant shaking. For flask growth an overnight culture of cells was diluted down to $\mathrm{A}_{600}$ of 0.1 and grown in $\mathrm{SC}$ media until an $\mathrm{A}_{600}$ of 0.4-0.5. Cells were pelleted and then resuspended in SC media lacking only the specified amino acid and growth was monitored for up to $24 \mathrm{~h}$.

\section{GCN4-lacZ reporter assay}

WY523 and WY525 ( $g c n 2 \Delta)$ cells were derived from strain EG328-1A transformed with plasmid p180, encoding GCN4-lacZ with the full complement of upstream ORFs [14]. Cells were grown overnight in SC media containing all amino acids. Cells were diluted down to $\mathrm{A}_{600} \sim 0.1$, then grown to $\mathrm{A}_{600} \sim 0.4-0.5$ at which point they were spun down and resuspended in media lacking the specified amino acid. $35 \mathrm{ml}$ cells were taken at designated time points and lysed using beads in 0.4 $\mathrm{ml}$ of $100 \mathrm{mM}$ Tris- $\mathrm{HCl} \mathrm{pH} \mathrm{8,20 \%} \mathrm{glycerol,} 1 \mathrm{mM} \beta$ -mercaptoethanol and $100 \mu \mathrm{M}$ PMSF. Lysate was clarified with a $10 \mathrm{~min}$ spin at 18,600 RFC. $20 \mu \mathrm{l}$ of extract was combined with $980 \mu \mathrm{l}$ of reaction buffer $(100 \mathrm{mM}$ Sodium Phosphate, $\mathrm{pH}$ 7.5, $10 \mathrm{mM} \mathrm{KCl,} 2 \mathrm{mM} \mathrm{MgSO}_{4}$, $4.5 \mathrm{mM} \beta$-mercaptoethanol). $200 \mu \mathrm{l}$ of o-nitrophenyl- $\beta$ D-galactopyranoside solution $(4 \mathrm{mg} / \mathrm{ml})$ was added and the reaction was allowed to proceed for 20 and $60 \mathrm{~min}$ at $28^{\circ} \mathrm{C}$. Reaction was quenched by adding $0.5 \mathrm{ml}$ of 1 $\mathrm{M} \mathrm{Na} \mathrm{CO}_{3}$ and absorbance was taken at $420 \mathrm{~nm}$.

\section{Genome-wide tRNA charging profile by microarrays}

Isolation, genome-wide profiling of charged tRNA was carried out as previously described [10] and in a Method video article [32]. The microarray data has been deposited in Geo data base (accession number GSE22452).

\section{Genome-wide tRNA abundance profile by microarrays}

the detailed procedures used for profiling tRNA abundance have been described previously [33,34].

\section{List of abbreviations}

GAAC: general amino acid control.

\section{Acknowledgements}

This work was supported in part by an exploratory grant from Ajinomoto Inc., Japan (to TP), by National Institutes of Health Grant R01GM49164 (to RCW), and by the NIH Training Grant T32GM007183-33 (to JZ). We thank Dr. Alan Hinnebusch for helpful discussions and suggesting the triple amino acid depletion experiment.

\section{Author details}

${ }^{1}$ Department of Biochemistry and Molecular Biology, University of Chicago, Chicago, IL 60637, USA. '2Department of Biochemistry and Molecular Biology, Indiana University School of Medicine, Indianapolis, Indiana 46202, USA.

\section{Authors' contributions}

JZ carried out the growth studies and microarray analysis. XCW assisted with the growth studies and performed some microarray analysis. RW participated in design of the study. TP participated in the design of the study and the preparation of the manuscript. All authors read and approved the final manuscript.

Received: 28 April 2010 Accepted: 4 August 2010

Published: 4 August 2010

\section{References}

1. Hinnebusch AG: Translational regulation of GCN4 and the general amino acid control of yeast. Annu Rev Microbiol 2005, 59:407-450.

2. Wek RC, Jiang H-Y, Anthony TG: Coping with stress: elF2 kinases and translational control. Biochem Soc Trans 2006, 34:7-11.

3. Sonenberg N, Hinnebusch AG: Regulation of translation initiation in eukaryotes: mechanisms and biological targets. Cell 2009, 136:731-745.

4. Harding HP, Zhang Y, Zeng H, Novoa I, Lu PD, Calfon M, Sadri N, Yun C, Popko B, Paules R, Stojdl DF, Bell JC, Hettmann T, Leiden JM, Ron D: An integrated stress response regulates amino acid metabolism and resistance to oxidative stress. Mol Cell 2003, 11:619-633.

5. Vattem KM, Wek RC: Reinitiation involving upstream ORFs regulates ATF4 mRNA translation in mammalian cells. Proc Natl Acad Sci USA 2004, 101:11269-11274.

6. Marciniak SJ, Ron D: Endoplasmic reticulum stress signaling in disease. Physiol Rev 2006, 86:1133-1149.

7. Wek RC, Jackson BM, Hinnebusch AG: Juxtaposition of domains homologous to protein kinases and histidyl-tRNA synthetases in GCN2 protein suggests a mechanism for coupling GCN4 expression to amino acid availability. Proc Natl Acad Sci USA 1989, 86:4579-4583.

8. Dong J, Qiu H, Garcia-Barrio M, Anderson J, Hinnebusch AG: Uncharged tRNA Activates GCN2 by Displacing the Protein Kinase Moiety from a Bipartite tRNA-Binding Domain. Molecular Cell 2000, 6:269-279.

9. Wek SA, Zhu S, Wek RC: The histidyl-tRNA synthetase-related sequence in the elF-2 alpha protein kinase GCN2 interacts with tRNA and is required for activation in response to starvation for different amino acids. Mol Cell Biol 1995, 15:4497-4506. 
10. Zaborske JM, Narasimhan J, Jiang L, Wek SA, Dittmar KA, Freimoser F, Pan T, Wek RC: Genome-wide analysis of tRNA charging and activation of the elF2 kinase Gcn2p. J Biol Chem 2009, 284:25254-25267.

11. Braus GH: Aromatic amino acid biosynthesis in the yeast Saccharomyces cerevisiae: a model system for the regulation of a eukaryotic biosynthetic pathway. Microbiol Rev 1991, 55:349-370.

12. Messenguy F, Dubois E: Regulation of Arginine Metabolism in Saccharomyces cerevisiae: a Network of Specific and Pleiotropic Proteins in Response to Multiple Environmental Signals. Food Technol Biotechnol 2000, 38:277-285.

13. Ramos F, Thuriaux P, Wiame JM, Bechet J: The participation of ornithine and citrulline in the regulation of arginine metabolism in Saccharomyces cerevisiae. Eur J Biochem 1970, 12:40-47.

14. Narasimhan J, Staschke KA, Wek RC: Dimerization is required for activation of elF2 kinase Gcn2 in response to diverse environmental stress conditions. J Biol Chem 2004, 279:22820-22832.

15. Hinnebusch $A G$, Fink GR: Positive regulation in the general amino acid control of Saccharomyces cerevisiae. Proc Natl Acad Sci USA 1983, 80:5374-5378.

16. Giaever G, Chu AM, Ni L, Connelly C, Riles L, Veronneau S, Dow S, LucauDanila A, Anderson K, Andre B, Astromoff A, El-Bakkoury M, Bangham R, Benito R, Brachat S, Campanaro S, Curtiss M, Davis K, Deutschbauer A, Entian KD, Flaherty P, Foury F, Garfinkel DJ, Gerstein M, Gotte D, Güldener U, Hegemann JH, Hempel S, Herman Z, Jaramillo DF, et al: Functional profiling of the Saccharomyces cerevisiae genome. Nature 2002, 418:387-391.

17. Niederberger $P$, Miozzari G, Hutter R: Biological role of the general control of amino acid biosynthesis in Saccharomyces cerevisiae. Mol Cell Biol 1981, 1:584-593.

18. Dever TE, Yang W, Astrom S, Bystrom AS, Hinnebusch AG: Modulation of tRNA(iMet), elF-2, and elF-2B expression shows that GCN4 translation is inversely coupled to the level of elF-2.GTP.Met-tRNA(iMet) ternary complexes. Mol Cell Biol 1995, 15:6351-6363.

19. Luttik MAH, Vuralhan Z, Suir E, Braus GH, Pronk JT, Daran JM: Alleviation of feedback inhibition in Saccharomyces cerevisiae aromatic amino acid biosynthesis: Quantification of metabolic impact. Metabolic Engineering 2008, 10:141-153.

20. Harbison CT, Gordon DB, Lee TI, Rinaldi NJ, Macisaac KD, Danford TW, Hannett NM, Tagne J-B, Reynolds DB, Yoo J, Jennings EG, Zeitlinger J, Pokholok DK, Kellis M, Rolfe PA, Takusagawa KT, Lander ES, Gifford DK, Fraenkel E, Young RA: Transcriptional regulatory code of a eukaryotic genome. Nature 2004, 431:99-104.

21. Paravicini $G$, Mosch HU, Schmidheini T, Braus $G$ : The general control activator protein GCN4 is essential for a basal level of ARO3 gene expression in Saccharomyces cerevisiae. 1989, 9:144-151.

22. Whitney PA, Morris DR: Polyamine auxotrophs of Saccharomyces cerevisiae. J Bacteriol 1978, 134:214-220.

23. Feller A, Dubois E, Ramos F, Pierard A: Repression of the genes for lysine biosynthesis in Saccharomyces cerevisiae is caused by limitation of Lys14-dependent transcriptional activation. Mol Cell Biol 1994, 14:6411-6418.

24. Thomas D, Surdin-Kerjan Y: Metabolism of sulfur amino acids in Saccharomyces cerevisiae. 1997, 61:503-532.

25. Chin CS, Chubukov V, Jolly ER, DeRisi J, Li H: Dynamics and design principles of a basic regulatory architecture controlling metabolic pathways. PLOS Biol 2008, 6:e146.

26. El Alami M, Dubois E, Oudjama Y, Tricot C, Wouters J, Stalon V, Messenguy F: Yeast Epiarginase Regulation, an Enzyme-Enzyme Activity Control. Journal of Biological Chemistry 2003, 278:21550-21558.

27. Chan PY, Cossins EA: Arginine metabolism in Saccharomyces cerevisiae. Some general properties of yeast arginase. 1973, 14:641-651.

28. Lanker S, Bushman JL, Hinnebusch AG, Trachsel H, Mueller PP: Autoregulation of the yeast lysyl-tRNA synthetase gene GCD5/KRS1 by translational and transcriptional control mechanisms. Cell 1992, 70:647-657.

29. Narasimhan J, Staschke KA, Wek RC: Dimerization is required for activation of elF2 kinase Gcn2 in response to diverse environmental stress conditions. J Biol Chem 2004, 279:22820-22832.

30. Yang R, Wek SA, Wek RC: Glucose limitation induces GCN4 translation by activation of Gen2 protein kinase. Mol Cell Biol 2000, 20:2706-2717.
31. Kaiser C, Michaelis S, Mitchell A: Methods in Yeast Genetics. Plainview, NY: Cold Spring Harbor Laboratory Press 1994, 207-217.

32. Zaborske J, Pan T: Genome-wide analysis of aminoacylation (charging) levels of tRNA using microarrays. J Vis Exp 2010 [http://www.jove.com/ index/Details.stp?|D=2007].

33. Dittmar KA, Goodenbour JM, Pan T: Tissue-Specific Differences in Human Transfer RNA Expression. PLoS Genet 2006, 2:e221.

34. Pavon-Eternod M, Gomes S, Geslain R, Dai Q, Rosner MR, Pan T: tRNA overexpression in breast cancer and functional consequences. Nucleic Acids Res 2009, 37:7268-7280.

doi:10.1186/1471-2091-11-29

Cite this article as: Zaborske et al: Selective control of amino acid metabolism by the GCN2 elF2 kinase pathway in Saccharomyces cerevisiae. BMC Biochemistry 2010 11:29.

\section{Submit your next manuscript to BioMed Central and take full advantage of:}

- Convenient online submission

- Thorough peer review

- No space constraints or color figure charges

- Immediate publication on acceptance

- Inclusion in PubMed, CAS, Scopus and Google Scholar

- Research which is freely available for redistribution

Submit your manuscript at www.biomedcentral.com/submit
C) Biomed Central 